\title{
Estimation of Surgical Needle Insertion Force Using Kalman Filter
}

\author{
Syed Riaz un Nabi Jafri, Ali Jamshaid, Syed Minhaj un Nabi Jafri, Jamshed Iqbal
}

\begin{abstract}
This paper presents a novel low-cost technique to measure the insertion force of a surgical needle on a testing surface to check needle strength. A combination of a load cell with a current sensor for a linear DC motor has been used to estimate the insertion force based on Kalman Filter (KF). The custom-designed and in-house fabricated system to estimate the insertion force comprises of the motor coupled with a vertically articulated arm. The needle to be tested is mounted at the end of the arm. Movement of the arm has been controlled electronically to produce the insertion force by the needle to a testing surface. The sensory measurement data generated during this process has been collected using Arduino based embedded electronic hardware. The $\mathrm{KF}$ based proposed strategy has been validated using the developed system by conducting various tests with different needles. Results in the form of penetration force and friction force have been experimentally observed and are then compared with standard force meters. Comparative analysis witnesses efficiency of the proposed approach.
\end{abstract}

Keywords: Kalman filter, sensor fusion, biomedical system, surgical needle, load cell, current sensor

\section{Introduction}

Advances in various domains of engineering like electrical and mechanical together with developments in information technology are reshaping medical and biomedical sectors [1]. Surgical needles have been considered as the most important and valuable tool to conduct surgical operations [2]. These needles are strong unlike the sewing or stitching needles used in textile industry. They are manufactured using state-of-art engineering technologies to ensure reliability, precision and high quality [3]. There are three major components associated with manufacturing of a surgical needle [4]. First component is material of the needle such as Stainless Steel (ASTM grade: 304, 316 etc.) with various strengths. Second component is the dimension and shape of needle that is length (range: $15-60 \mathrm{~mm}$ ) and diameter (range: 0.3-1.6 mm) [5] as shown in Fig. 1. Third component is suture (thread) which can be classified as absorbable, non-absorbable and synthetic based on treatment area [6].

Surgeons have been trained for years to use surgical needles by applying proper hand motion in order to exert correct force on the needle to penetrate targeted skin while performing surgery. However, several concerns have been raised by surgeons or hospital administration about the quality of surgical needles. There is still an ample room for understanding of how surgical needles exert force on tissues of human body [7]. Straight body cutting needle with taper point is commonly used for stitches over the skin [8]. During clinical procedures, the clinician, needle and patient are all part of a complex dynamical system with many interactions. Considering manual needle insertion during a clinical procedure, a reported study [9] presents force versus position profile and the corresponding velocity versus position diagram.

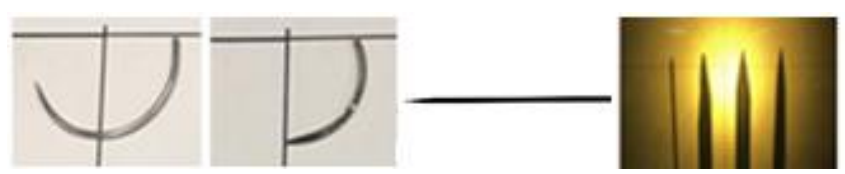

(a)

(b)

(c)

(d)

Fig. 1. Multiple types of surgical needles and their tips (a) $1 / 2$ Circle (b) $1 / 4$ Circle (c) Straight (d) Magnified view

Globally a number of testing solutions have been developed for estimation of needle insertion force for quality checks [9][10]. Microsharp needles Ltd. has used optical magnifiers to visualize needles tip in order to qualify them for surgical operation [11]. Ethicon Inc. has developed an automated robotic 3D force measurement arm with the capability of using multi-specimen bed for insertion test of single surgical needle in their manufacturing process. Another company I\&Z has designed a lab testing equipment to measure needle penetration force along Z-axis. Instron has developed rotational robotic arm based testing equipment for the measurement of needle penetration force.

Researchers have proposed several models of a soft tissue to investigate the needle insertion force with automated surgical needle suturing [12]. Naemura et al. analyzed the impact of force-position curve just after puncture due to variations in tip shape and generated slope [13]. Results have been presented by considering insertion of a Tuohy needle through silicone rubber membranes at a 
velocity of $2 \mathrm{~mm} / \mathrm{sec}-8 \mathrm{~mm} / \mathrm{sec}$. Another research [14] has explained the effect of velocity on insertion force for a straight $(0.88 \mathrm{~mm})$ suture needle which has been inserted into sheep skin at different skin-tension levels. In addition to the above-mentioned research findings, surgical trends are rapidly moving towards robotically assisted minimally invasive surgery (RAMIS) [15]. Researchers have used Da Vinci robotic kit and proposed closed loop control for simulating minimally invasive suturing [16]. Similarly, Xiao et al. designed a robotic prototype for surgical needle insertion integrated with fibre optic force sensor and reported insertion force results [17]. Another research study [18] presented a model of needle tissue inter-linkage while analyzing tissue puncturing as a function of tissue deformation using a 6 DOF force sensor.

In developing countries with greater population like Pakistan, it is a big challenge to provide assured quality controlled surgical needles to well-trained surgeons. There are around 117 medical colleges, 1169 big hospitals and thousands of small scale hospitals in the country performing major/minor surgeries. In order to check the quality of needle, the common conventional practice is to penetrate the needle manually into a thick card paper $15-20$ times. Later on, needle tip has been inspected manually through two hundred times magnification. Recently, few advanced companies have introduced reliable testing services based on the standard force measuring meters like INSIZE ISF-50N as shown in Fig. 2(a). It is capable of measuring $\pm 50 \mathrm{~N}$ in compression or tension mode. As illustrated in Fig. 2(b), a peak force is observed when the surgical needle penetrates into the tissue. This force then reduces sharply and the frictional force retains as penetration depth increases w.r.t. time [19]. Due to high cost of the force meter, most of the local medical entities are unable to utilize it thereby needing a cheaper in-house solution for quality assurance.

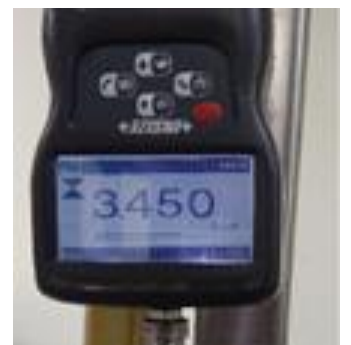

(a)

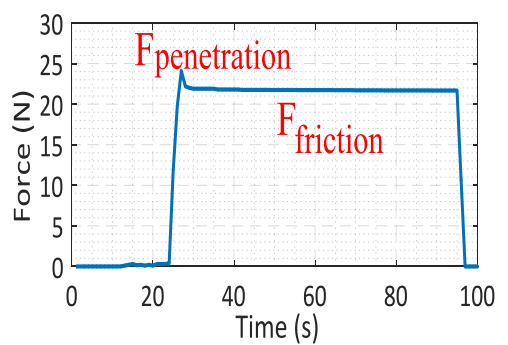

(b)
Fig. 2. (a) INSIZE ISF-50N force meter with needle gripper (b) Penetration force graph versus time

The novelty of the present research work lies in proposing cascaded Kalman Filter (KF) based estimation technique using measurements from low-cost load cells and current sensor when compared with existing solutions of relatively higher cost [17]. Additionally this work proposes a unique low-cost hardware design to estimate the insertion force of a surgical needle by incorporating required instrumentation. The remaining of the paper is organized as follows: Section 2 briefly describes mechanical model and portrays custom designed prototyped system. Instrumentation hardware design has presented in Section 3. Calibration of sensors is discussed in Section 4. Section 5 presents KF design and experimental results with surgical needles and finally Section 6 concludes the paper.

\section{Mechanical Model}

The custom-made hardware for measuring the insertion force of a needle is based on mechanical CAD model designed in SolidWorks, which is illustrated in Fig. 3(a). To generate a controlled penetration movement of the needle, a DC linear actuator, installed on top of a tower, moves an arm in Z-axis. This arm holds force meter and the needle at its end. The $400 \mathrm{~mm}$ high vertical tower is made up of stainless steel sheet of thickness $1.5 \mathrm{~mm}$. It is welded with $130 \mathrm{~mm}$ high iron-made unit which houses the electronic circuitry along with driver modules. A specialized needle gripper is designed and is integrated with the force meter in order to provide flexibility of gripping several needles of various dimensions. The fabricated prototype is shown in Fig. 3(b).

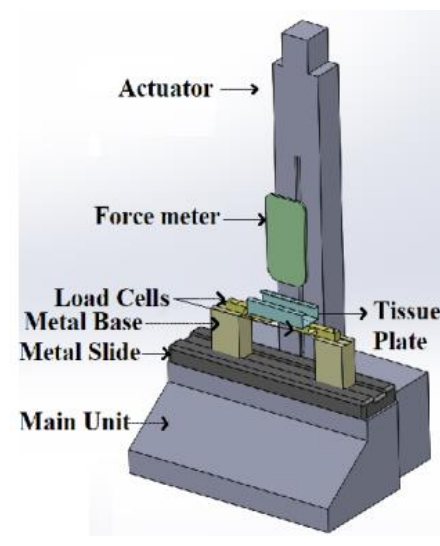

(a)

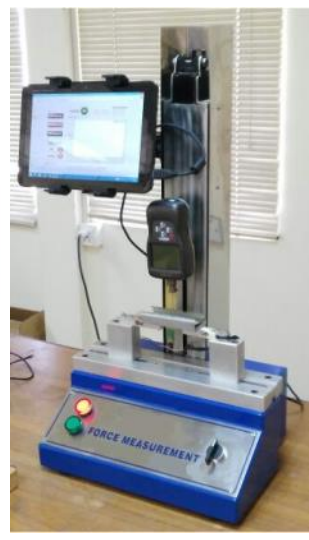

(b)
Fig. 3. (a) SolidWorks mechanical model

(b) Fabricated prototype

\section{Instrumentation Hardware Design}

Design of instrumentation circuitry involves two stages: First stage deals with smooth operation of the actuator as per operator's desired settings. Second stage is the sensing circuitry using load cells, current sensor and position sensor. 
Both stages have been interfaced with Arduino UNO (ATMega328 microcontroller) embedded board as shown in Fig. 4. For the first stage, based on a user's preferred settings, Arduino board generates commands sent to relay module to smoothly run the actuator until it has commanded to stop. During motion of the actuator, position of the arm (hence testing needle) has been sensed by a position sensor which is interfaced with Arduino. At the same time, current value of the actuator (as a function of bearing load) has been sensed through a current sensor. Penetration force data is acquired through two load cells and complete data profile has been finally sent to Laptop/HMI device after every $90 \mathrm{msec}$.

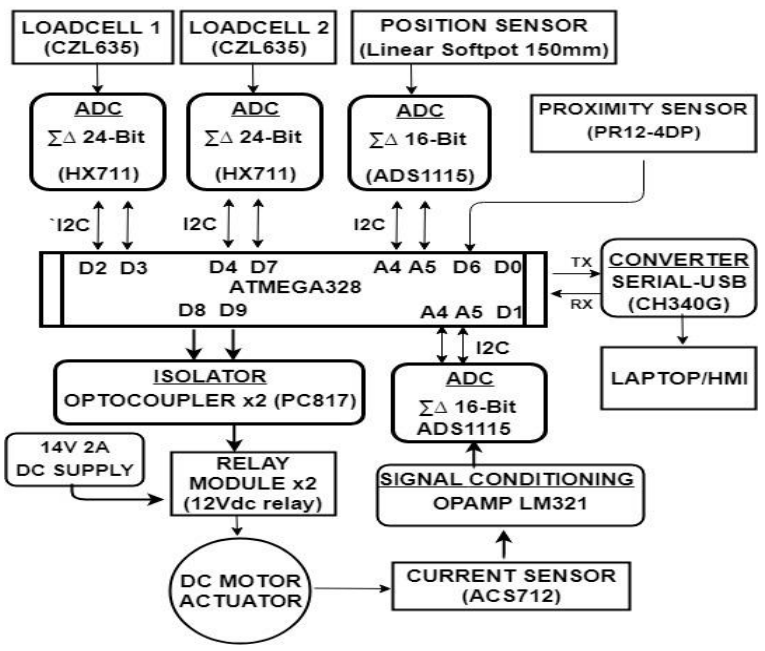

Fig. 4. Block diagram of the instrumentation circuitry

\subsection{Force Measurement using Load Cells}

A load cell is a force detecting module having strain gauges mounted in the structure. The electrical signal acquired by a load cell is very small and requires amplification. The capacity of the load cell used in the present research is $20 \mathrm{~kg}$ and it is connected to the Arduino board through HX711 $\mathrm{ADC}$ as shown in figure 5. Software based I2C protocol has been implemented to read load cell measurements. The second load cell with the same specifications has been interfaced in a similar manner as depicted in Fig. 4.

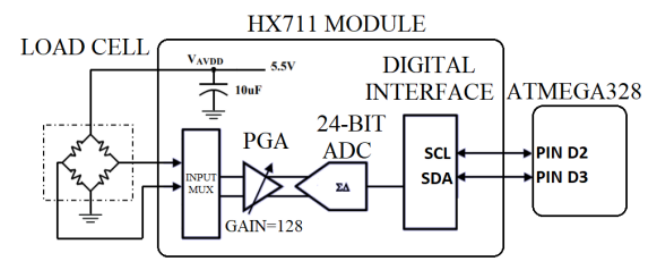

Fig. 5. Interface circuitry of load cell with Arduino

\subsection{Force Measurement Using Current Sensor}

To determine the load on the actuator, Hall effect based linear current sensor ACS712 has been integrated with Arduino through ADC module (ADS1115) board as shown in figure 6 . The input measuring range of current sensor is $\pm 5 \mathrm{~A}$ and the analog output of the sensor is $1.5 \mathrm{~V}-3.5 \mathrm{~V}$. Based on signal conditioning, the desired voltage output has been set and fed to ADC which communicates with the Arduino board through I2C protocol.

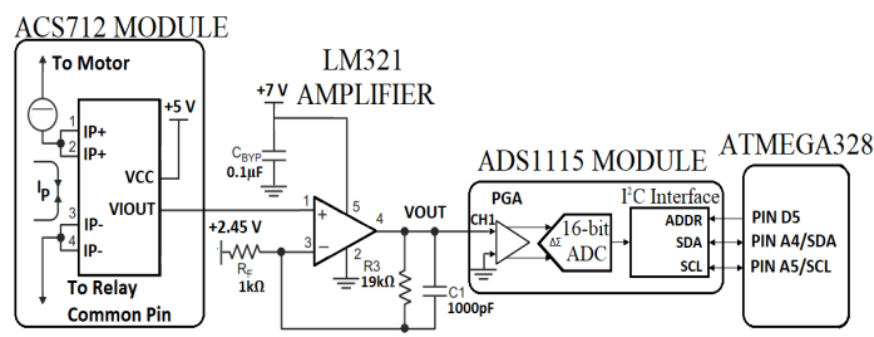

Fig. 6. Interfacing circuitry of current sensor with Arduino

\subsection{Driving Mechanism for Linear DC Motor}

DC gear motor coupled with the linear actuator of $150 \mathrm{~mm}$ travel length has been used to move the arm and to test the needle setup. It operates with a supply voltage of $13 \mathrm{~V}$ and a maximum current rating of $3 \mathrm{~A}$. In order to move in both up-down directions, input supply has been connected through SPDT relay which receives a command from Arduino as shown in Fig. 7. Current sensor coupled with the motor has attached to sense bearing load. Softpot linear position sensor has used for detecting position of the arm. For actuator's safety, proximity sensors have been attached at both ends of linear shaft and connected to Arduino.

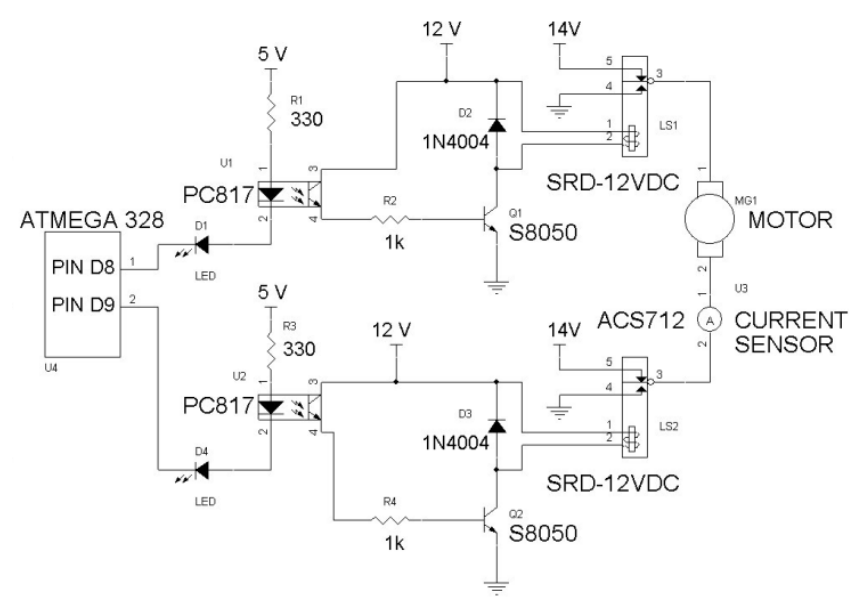

Fig. 7. Driving circuitry of linear DC motor

\section{Calibration of Sensors}

Performance of the proposed system is heavily dependent on calibration of the individual sensors. Calibration mechanism states the relationship of sensor's output signal to the input physical parameters input of the sensor. The calibration schemes of each sensor is discussed below:

\subsection{Calibration of Load Cells}

Calibration of independent load cell has been achieved by using standard weights ranging from $10 \mathrm{gm}$ to $2200 \mathrm{gm}$. 
Overall 10 samples of measurements have been taken for each standard weight of load cells as shown in Fig. 8(a). Complete results are presented in Table 1 and Table 2 for both cells. It is observed that standard deviation has been changed for different weights. However, in the present research, standard deviation of the highest weight has been considered which is also nearest to the datasheet. Load cell 2 appears to be more accurate than load cell 1 .

Table 1. Calibration of load cell 1

\begin{tabular}{|c|c|c|c|c|c|c|}
\hline No. & $\begin{array}{c}\text { Standard } \\
\text { Weight gm }\end{array}$ & $\begin{array}{c}\text { Test 1 } \\
\text { gm }\end{array}$ & $\begin{array}{c}\text { Test 2 } \\
\text { gm }\end{array}$ & $\begin{array}{c}\text { Test 10 } \\
\text { gm }\end{array}$ & $\begin{array}{c}\text { Mean } \\
\text { gm }\end{array}$ & $\begin{array}{c}\text { Standard } \\
\text { Deviation }\end{array}$ \\
\hline 1 & 10 & 6.2 & 8.6 & 7.5 & 7.4 & 1.2 \\
\hline 2 & 100 & 92.3 & 96.3 & 96.0 & 94.9 & 2.2 \\
\hline 3 & 2200 & 2191.6 & 2179.5 & 2198.2 & 2189.8 & 14.4 \\
\hline
\end{tabular}

Table 2. Calibration of load cell 2

\begin{tabular}{|c|c|c|c|c|c|c|}
\hline No. & $\begin{array}{c}\text { Standard } \\
\text { Weight gm }\end{array}$ & $\begin{array}{c}\text { Test 1 } \\
\text { gm }\end{array}$ & $\begin{array}{c}\text { Test 2 } \\
\text { gm }\end{array}$ & $\begin{array}{c}\text { Test 10 } \\
\text { gm }\end{array}$ & $\begin{array}{c}\text { Mean } \\
\text { gm }\end{array}$ & $\begin{array}{c}\text { Standard } \\
\text { Deviation }\end{array}$ \\
\hline 1 & 10 & 6.5 & 8.2 & 8.5 & 7.7 & 1.0 \\
\hline 2 & 100 & 100.2 & 99.3 & 102.1 & 100.5 & 1.4 \\
\hline 3 & 2200 & 2207.3 & 2211.8 & 2196.3 & 2205.8 & 10.3 \\
\hline
\end{tabular}

In the next phase, calibration of both load cells has been carried out by mounting base plate which holds standard weights. In this way, actual penetration measurements have been observed as shown in figure 8(b). Observed results have been shown in table 3 . It is evident from the practical results that the force inserting on the base plate (on top of load cells) is unequally divided between both load cells. Various standard weights have been utilized to determine percentage of force distribution. Approximate distribution has been found to be $46 \%$ and $54 \%$ for load cell 1 and 2 respectively using least square fitting. It is evident from Table 3 that both load cells exhibit deviation to expected value because of inaccuracies, with load cell 2 demonstrating more accuracy compared with load cell 1 .

Table 3. Integrated calibration of load cells

\begin{tabular}{|c|c|c|c|}
\hline No. & $\begin{array}{c}\text { Standard } \\
\text { Weight gm }\end{array}$ & $\begin{array}{c}\text { Load Cell 1 } \\
\text { gm }\end{array}$ & $\begin{array}{c}\text { Load Cell 2 } \\
\text { gm }\end{array}$ \\
\hline 1 & 10 & 4.6 & 5.5 \\
\hline 2 & 100 & 48 & 54 \\
\hline 3 & 2200 & 1022 & 1194 \\
\hline
\end{tabular}

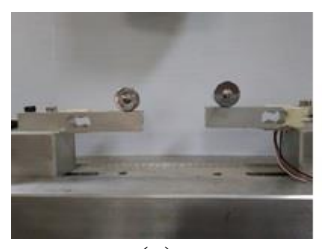

(a)

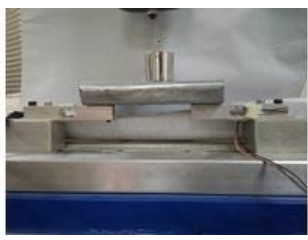

(b)

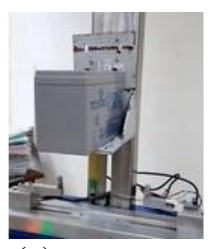

(c)
Fig. 8. Calibration of load cells and current sensor (a) Independent calibration of load cells (b) Integrated calibration of load cells (c) Calibration of current sensor

\subsection{Calibration of Current Sensor}

In order to determine load carrying characteristics of the DC motorized linear actuator, a series of known weights has been utilized to determine current flow as shown in figure 8 (c). It is observed that the actuator is sinking $0.25 \mathrm{~A}$ at $14 \mathrm{~V}$ with no load attached to its shaft. However, with variation of weights, the amount of current changes almost linearly with the rotational speed kept as constant. Therefore, a number of known weights (e.g. a battery of $2200 \mathrm{gm}$ ) has been utilized to determine relationship between weight and current using least square fitting. For each weight, ten samples have been recorded by moving actuator downside as shown in Table 4. The maximum value of standard deviation has been used in the present work.

Table 4. Calibration of current sensor

\begin{tabular}{|c|c|c|c|c|c|c|}
\hline No. & $\begin{array}{c}\text { Known } \\
\text { Weight } \\
\text { gm }\end{array}$ & $\begin{array}{c}\text { Test 1 } \\
\text { gm }\end{array}$ & $\begin{array}{c}\text { Test 2 } \\
\text { gm }\end{array}$ & $\begin{array}{c}\text { Test 10 } \\
\text { gm }\end{array}$ & $\begin{array}{c}\text { Mean } \\
\text { gm }\end{array}$ & $\begin{array}{c}\text { Standard } \\
\text { Deviation }\end{array}$ \\
\hline 1 & 2200 & 2196 & 2198 & 2201 & 2201.0 & 1.045 \\
\hline 2 & 3660 & 3662 & 3661 & 3662 & 3659.1 & 1.158 \\
\hline 3 & 5860 & 5858 & 5860 & 5858 & 5859.3 & 1.236 \\
\hline
\end{tabular}

\section{Kalman Filter Design}

Due to presence of noise in sensors measurements, KF has been used to estimate the penetration force [20]. KF has been utilized to minimize uncertainty in measurement of each sensor and to estimate the penetration force and finally to compare with the true value as observed through standard INSIZE force meter.

\subsection{Filtering of Discrete Sensors}

In order to measure the penetration force applied by the needle, the measurement of each sensor is first converted to a unit of force in $\mathrm{kgf}$ (kilogram force) where $1 \mathrm{kgf}$ is equal to the force applied by a $1 \mathrm{~kg}$ weight in the gravitational field of $g=9.8 \mathrm{~m} / \mathrm{s}^{2}$. A set of multiple measurements has been taken from these sensors corresponding to a specific input while observing the estimated KF. Following set of standard KF equations has been applied to determine the estimated output.

$$
\begin{gathered}
X_{P}=A X_{n-1}+B U_{n} \\
P_{p}=A P_{n-1} A^{T}+Q
\end{gathered}
$$

where $X_{p}$ is new predicted value of the variable to be estimated, $P_{p}$ is new predicted value of the variance of the variable, $U_{n}$ is Control vector (not used in this experiment), $\mathrm{A}$ is state transition coefficient (unity, in this case), B is control coefficient (zero, in this case) and Q is process error variance (small value has been considered). After determining prediction result from (1-2), a set of corrective equations has been applied to compute the estimated value 
as given in (3-4).

$$
\begin{aligned}
& X_{n}=X_{p}+K Y \\
& P_{n}=(1-K H) P_{P}
\end{aligned}
$$

where $X_{n}$ is new estimate of the variable for current measurement, $P_{n}$ is new estimate of variance of the variable, $\mathrm{H}$ is observation coefficient (unity in this case). The data acquired from the sensors includes; current measurement of variable $\left(Z_{n}\right)$ and measurement error variance i.e. standard deviation $(\mathrm{R})$.

The above algorithm has been applied to all individual sensors. Figure 9 shows the raw sensorial data acquired through load cell 1 under the application of $5 \mathrm{kgf}$ force and the filtered output received after KF estimation. As can be seen, the filtered output significantly improves the measurement noise of the sensor. Similar procedure has been applied to the measurements of other load cell and current sensor.

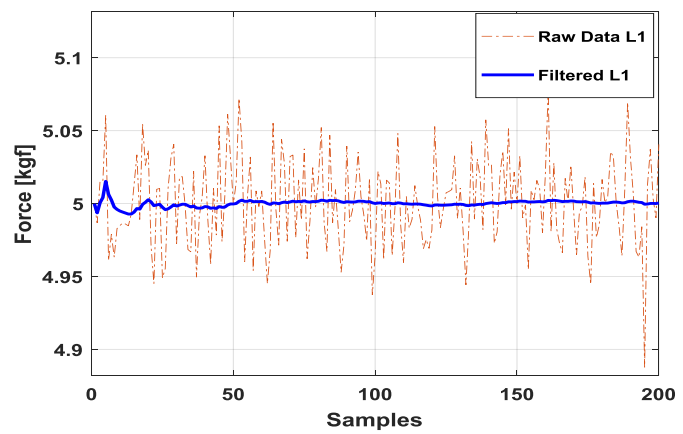

Fig. 9. Filtering of load cell 1 sensor through KF

\subsection{Cascaded Kalman Filter}

In order to utilize measurements of all sensors to estimate the penetration force, a cascaded KF architecture has been implemented as shown in Fig. 10.

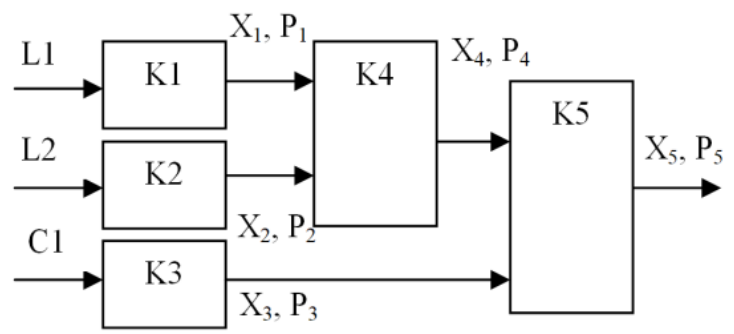

Fig. 10. Cascaded KF architecture

In this configuration, the inputs $\mathrm{L} 1, \mathrm{~L} 2$ and $\mathrm{C} 1$ are the measurements obtained from load cell 1 , load cell 2 and current sensor respectively. $\mathrm{K} 1, \mathrm{~K} 2$ and $\mathrm{K} 3$ are the respective single variable KFs applied on the measurement of the corresponding discrete sensor. $X_{i} \& P_{i}$ are the estimate for Ki. The fourth KF utilizes the output of K1 for prediction and output of $\mathrm{K} 2$ for correction and generates its estimate using measurements from both load cells. For prediction, the less accurate load cell has been used whereas the more accurate cell has been used for correction step. Similarly, the output of K4 is used in the prediction step of final filter $\mathrm{K} 5$, while the output of current sensor is used in the correction. The resultant output obtained through this configuration is shown in Fig. 11, where it can be seen that cascaded filtered output provides more accurate results compared to any of the individual sensor's results in response to a known applied force.

\subsection{Experimental Results}

Once the efficacy of the proposed cascaded KF has been established, real experiments have been conducted with two types of surgical needles; taper cut tip and round tip.

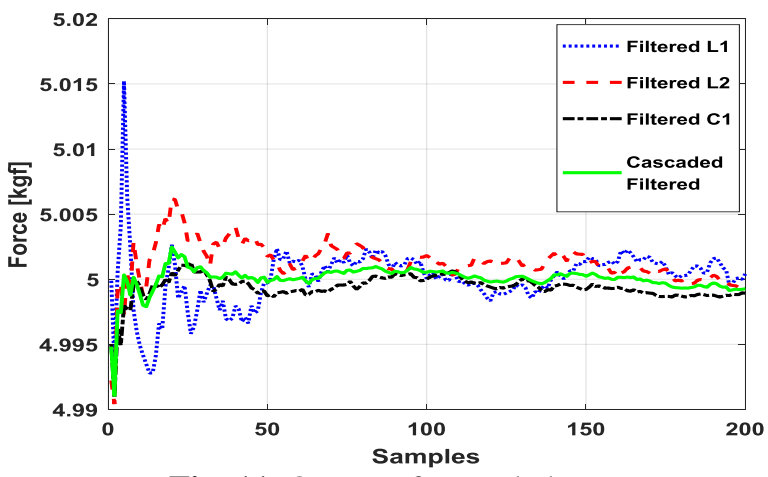

Fig. 11. Output of cascaded KF

The insertion force has been measured for tissues material. For the straight needle with taper cut tip of $1.0 \mathrm{~mm}$ diameter, the peak and stable readings corresponding to penetration force and insertion force are shown in Fig. 12.

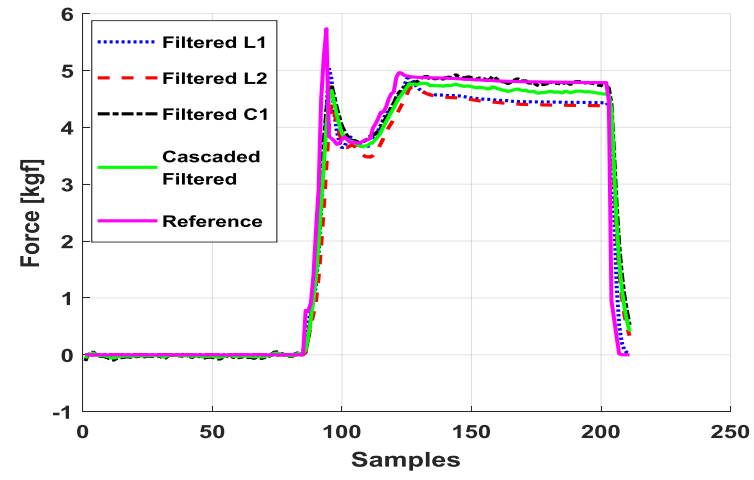

Fig. 12. Comparison of filtered results in case of $1.0 \mathrm{~mm}$ diameter taper cut tip needle

The result obtained are then compared with readings of a commercially available INSIZE force meter, which is considered as a reference The comparative analysis, given in Table 5, reveal that the proposed design provides fairly accurate measurement of the needle insertion force in comparison to the reference sensor both in the transient and steady state conditions. This is further confirmed by 
changing the diameter of taper cut tip needle. Figure 13 illustrates the comparative experimental results.

Table 5. Comparison of results for two surgical needles

\begin{tabular}{|c|c|c|c|c|}
\hline \multirow{2}{*}{ Needle type } & \multicolumn{2}{|c|}{$F_{\text {penetration }}(\mathrm{Kgf})$} & \multicolumn{2}{c|}{$\mathrm{F}_{\text {friction }}(\mathrm{Kgf})$} \\
\cline { 2 - 5 } & $\begin{array}{c}\text { Proposed } \\
\text { sys. }\end{array}$ & Ref. & $\begin{array}{c}\text { Proposed } \\
\text { sys. }\end{array}$ & Ref. \\
\hline Taper cut tip & 5.4 & 5.5 & 4.7 & 4.8 \\
\hline Round tip & 3.3 & 3.4 & 3.3 & 3.3 \\
\hline
\end{tabular}

The developed patterns of penetration forces followed by frictional forces shown in Fig. 12 and Fig. 13 depict better trend than the generated pattern reported in [17] (refer to figure 7 of this work) and further matches with the presented result in [18] (see figure 5 of this reference) showing the robustness of the estimation technique proposed in the present paper.

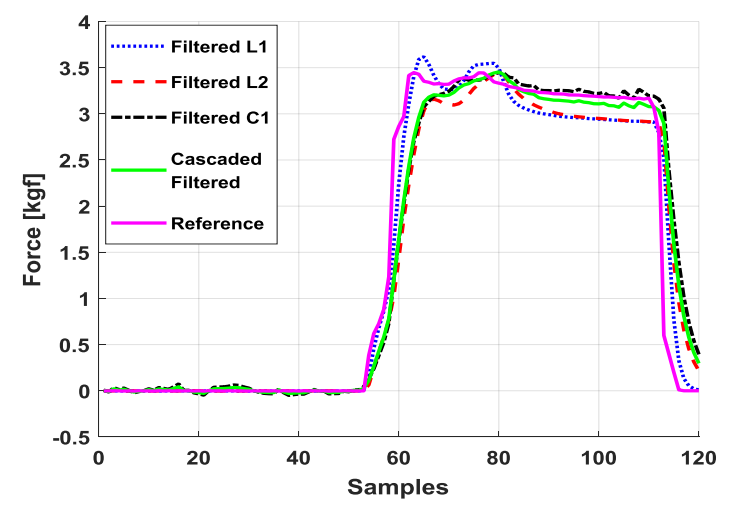

Fig. 13. Comparison of filtered results in case of $1.2 \mathrm{~mm}$ diameter taper cut tip needle

\section{Conclusion}

This paper proposes a novel technique to measure penetration and friction forces during insertion process of a surgical needle in order to check strength of needle tip. Various experimental tests have been conducted using straight needles with different diameters and different tip shapes. The proposed cascaded KF approach combines the advantages offered by the low-cost sensors and provides an optimized output when compared with an industry standard reference instrument. Comparative cost analysis revealed that the proposed system is six times cheaper than commercially available INSIZE ISF-50N force meter while still providing adequate accuracy, repeatability and reliability. Future work includes improving the prototype and conducting feasibility analysis for its commercialization.

\section{Acknowledgements}

The authors acknowledge the support of Ophth Pharma (Pvt. Ltd., Pakistan) for funding this research and providing hardware equipment and clinical assistance in testing the proposed estimation technique.

\section{References}

[1] S. Park, J. Kim, K. Ko, I. Jang and K. Sim, "Real-time heart rate monitoring system based on ring-type pulse oximeter sensor", Journal of Electrical Engineering and Technology, vol. 8, pp. 376-384, 2013.

[2] C. Yag-Howard, "Sutures, needles and tissue adhesives: a review for dermatologic surgery", Dermatologic Surgery, vol. 40, pp. S3-S15, 2014.

[3] C. Dennis, S. Sethu, S. Nayak, L. Mohan, Y.Y. Morsi and G. Manivasagam, "Suture materials - Current and emerging trends". Journal of Biomedical Materials Research, vol. 104, no. 6, pp. 1544-159, 2016

[4] Y. Cai, J. Moore, and Y. Lee, "Novel surgical needle design and manufacturing for vibratory-assisted insertion in medical applications", Computer-Aided Design and Applications, vol. 14, pp. 833-843, 2017.

[5] J. Smith and M. Macsai, "Needles, sutures and instruments", Ophthalmic Microsurgical Suturing Techniques, Springer, 2007, pp. 9-20.

[6] C. Dennis, S. Sethu, S. Nayak, L. Mohan, Y. Morsi, and G. Manivasagam, "Suture materials: Current and emerging trends", Journal of Biomedical Materials Research, vol. 104, pp. 1544-1559, 2016.

[7] D. Gerwen, J. Dankelman, and J. Dobbelsteen, "Needle-tissue interaction forces: A survey of experimental data", Medical Engineering and Physics, vol. 34, pp. 665-680, 2012.

[8] F. Selvi, S. Cakarer, T. Can, S. Topcu, A. Palancioglu and B. Keskin, "Effects of different suture materials on tissue healing", Journal of Istanbul University Faculty of Dentistry, vol. 50, pp. 35, 2016.

[9] D. Gerwen, "Needle-tissue interaction by experiment, PhD Thesis", TU Delft, Dec. 2013.

[10] A. Tahir, J. Iqbal, and T. Aized, "Human machine interface: Robotizing the instinctive living", Int Rob Auto J, vol. 4, pp. 308-314, 2018.

[11] Microsharp Pvt. Ltd., "Manufacturer of suture needles", www.microsharpneedles.com.

[12] N. Abolhassani, R. Patel, and M. Moallem, "Needle insertion into soft tissue: A survey", Medical Engineering and Physics, vol. 29, pp. 413-431, 2007.

[13] K. Naemura, A. Sakai, T. Hayashi, and H. Saito, "Epidural insertion simulator of higher insertion resistance \& drop rate after puncture", IEEE International Conference of Engineering in Medicine and Biology Society, 2008, pp. 3249-3252.

[14] T. Frick, D. Marucci, J. Cartmill, C. Martin, and W. Walsh, "Resistance forces acting on suture needles", Journal of Biomechanics, vol. 34, pp.1335-1340, 2001

[15] R. Jackson, V. Desai, J. Castillo, and M. Çavuşoğlu, "Needle-tissue interaction force state estimation for robotic surgical suturing", IEEE/RSJ International 
Conference on Intelligent Robots and Systems, 2016.

[16] F. Zhong, Y. Wang, Z. Wang and Y. Liu , "Dual-arm robotic needle insertion with active tissue deformation for autonomous suturing", IEEE Robotics and Automation Letters, vol. 4, no. 3, pp. 2669 - 2676, 2019.

[17] L. Xiao, T. Yang, B. Huo, X. Zhao, J. Han and W. Xu, "Impedance control of a robot needle with a fiber optic force sensor", IEEE International Conference on Signal Processing, Chengdu, China, 2016, pp. 13791383

[18] M. Khadem, C. Rossa, R. Sloboda, N. Usmani and M. Tavakoli, "Mechanics of tissue cutting during needle insertion in biological tissue," IEEE Robotics and Automation Letters, vol. 1, no. 2, pp. 800-807, 2016.

[19] L. Xiao, T. Yang, B. Huo, X. Zhao, J. Han, and W. Xu, "Impedance control of a robot needle with a fiber optic force sensor", Chengdu, China, IEEE International Conference on Signal Processing, 2016, pp. 1379-1383.

[20] P. Kim, "Kalman filter for beginners: with MATLAB examples", CreateSpace Independent Publishing Platform, ISBN: 978-1463648350, 2011. 